\title{
Screening of Antioxidant Properties of the Apple Juice Using the Front-Face Synchronous Fluorescence and Chemometrics
}

\author{
Katarzyna Włodarska ${ }^{1}$ - Katarzyna Pawlak-Lemańska ${ }^{1}$ • Igor Khmelinskii ${ }^{2}$. \\ Ewa Sikorska ${ }^{1}$
}

Received: 21 May 2016 / Accepted: 7 November 2016 / Published online: 16 November 2016

(C) The Author(s) 2016. This article is published with open access at Springerlink.com

\begin{abstract}
Fluorescence spectroscopy is gaining increasing attention in food analysis due to its higher sensitivity and selectivity as compared to other spectroscopic techniques. Synchronous scanning fluorescence technique is particularly useful in studies of multi-fluorophoric food samples, providing a further improvement of selectivity by reduction in the spectral overlapping and suppressing light-scattering interferences. Presently, we study the feasibility of the prediction of the total phenolics, flavonoids, and antioxidant capacity using front-face synchronous fluorescence spectra of apple juices. Commercial apple juices from different product ranges were studied. Principal component analysis (PCA) applied to the unfolded synchronous fluorescence spectra was used to compare the fluorescence of the entire sample set. The regression analysis was performed using partial least squares (PLS1 and PLS2) methods on the unfolded total synchronous and on the single-offset synchronous fluorescence spectra. The best calibration models for all of the studied parameters were obtained using the PLS1 method for the single-offset synchronous spectra. The models for the prediction of the total flavonoid content had the best performance; the optimal model was obtained for the analysis of the synchronous fluorescence spectra at $\Delta \lambda=110 \mathrm{~nm}\left(R^{2}=0.870\right.$, residual predictive deviation $(\mathrm{RPD})=2.7)$. The optimal calibration models for the
\end{abstract}

Ewa Sikorska

ewa.sikorska@ue.poznan.pl

1 Faculty of Commodity Science, Poznań University of Economics and Business, al. Niepodległości 10, 61-875 Poznań, Poland

2 Universidade do Algarve, FCT, DQF and CIQA, Campus de Gambelas, 8005-139 Faro, Portugal prediction of the total phenolic content $(\Delta \lambda=80 \mathrm{~nm}$, $\left.R^{2}=0.766, \mathrm{RPD}=2.0\right)$ and the total antioxidant capacity $\left(\Delta \lambda=70 \mathrm{~nm}, R^{2}=0.787, \mathrm{RPD}=2.1\right)$ had only an approximate predictive ability. These results demonstrate that synchronous fluorescence could be a useful tool in fast semiquantitative screening for the antioxidant properties of the apple juices.

Keywords Apple juice · Total phenolics · Total flavonoids · Synchronous fluorescence $\cdot$ Partial least squares regression

\section{Introduction}

Phenolic compounds account for the metabolic activity and antioxidant properties of plant-based foods and for the putative health benefits to humans (Scalbert et al. 2005). Moreover, polyphenols affect organoleptic properties of foods: their color, flavor, astringency, and hardness (Robbins 2003). The assessment of polyphenolic compounds and their antioxidant activity is one of important aspects of quality studies of many foods.

The HPLC method is mainly used for the assessment of individual phenolics, coupled with absorbance, mass spectrometric, or fluorimetric detection (Corradini et al. 2011; Ignat et al. 2011; Pyrzynska and Sentkowska 2014).

Spectrophotometric methods based on specific reactions are commonly used for the analysis of the overall antioxidant contents, or of specific classes of antioxidants. In particular, the Folin-Ciocalteu assay is widely used for determining the total phenolics. The total flavonoid contents may be determined using a colorimetric method based on the complexation with Al(III) (Ignat et al. 2011).

The spectrophotometric assays of the total antioxidant capacity of foods are usually based on comparing the sample 
ability to scavenge free radicals or reduce other chemicals to that of a reference compound (Craft et al. 2012).

Spectroscopic methods coupled with chemometrics provide an alternative to the conventional measurements, particularly in the screening or/and routine analysis. They are widely used in the assessment of food quality. The spectra serve as the product fingerprints in such applications. The relationship between the spectra and the qualitative or quantitative product properties is established using either multivariate classification or regression methods. The multivariate models, once developed, may be used to predict the properties of new samples by their spectra. The measurements are simple and fast and may be performed on samples directly without pretreatment or using chemical reagents. This feature enhances the labor-, time-, and cost-effectiveness of the analysis. The procedure is environmentally friendly and complies with the requirements for the green analytical chemistry (Armenta et al. 2008).

Quantification of the bioactive compounds and functional properties by use of spectroscopy is one of the important application areas for these methods. The application of infrared spectroscopy combined with chemometrics for determination of the antioxidant contents and antioxidant capacity has been recently reviewed (Cozzolino 2015; Lu and Rasco 2011).

The vibrational spectra in the near- (NIR) and mid-infrared (MIR) ranges provide structural information about the organic components and thus are used for quantifying both major and minor food constituents (Cozzolino 2015; Nicolaï et al. 2007). Depending on the complexity of the food matrix, chemical character, and concentration of the analyte, the sensitivity of these techniques to minor constituents may be quite low. This problem is especially frequent in plant-based foods having water as the main constituent, which dominates the NIR and MIR absorption spectra. Raman spectroscopy based on inelastic light scattering provides spectral information complementary to MIR spectroscopy and eliminates water interferences; however, it is limited by the low signal intensity and interferences from sample fluorescence (Lohumi et al. 2015).

Recent studies indicate that fluorescence may be an alternative to the vibrational spectroscopic techniques in studying the antioxidant abundance and antioxidant capacity in food matrices (Monago-Maraña et al. 2016; Orzel and Daszykowski 2014; Orzel et al. 2015; Žiak et al. 2014). The main advantages of fluorescence as compared to other spectroscopic techniques are its high sensitivity and selectivity. Due to these features, fluorescence may be especially useful in studies of minor components in complex food matrices and in the presence of interferences (Christensen et al. 2006). Moreover, fluorescence spectra should be easier to interpret. However, this technique is restricted to fluorescent components. Various emission properties of the food antioxidants may also pose additional limitations. For example, ascorbic acid is not fluorescent and tocopherols are highly fluorescent, while phenolic compounds have very diverse fluorescence properties (Christensen et al. 2006, Mazina et al. 2015; Wolfbeis et al. 1984).

The overall emission of complex, multi-constituent samples is usually characterized by the total fluorescence spectra, called also the excitation-emission matrices (EEMs) (Christensen et al. 2006). They are obtained by recording the emission spectra for a series of excitation wavelengths, thus providing comprehensive characteristics of the absorption and fluorescence properties of all the emitting components in the samples studied.

Synchronous fluorescence spectroscopy is another technique used for the complex food samples. Here, fluorescence intensity is measured as a function of the simultaneously scanned emission and excitation wavelengths, usually with a constant offset between them $\left(\Delta \lambda=\lambda_{\mathrm{em}}-\lambda_{\mathrm{ex}}\right)$. The main advantages of the synchronous fluorescence spectra (SyF) as compared to the EEMs are their improved selectivity and sensitivity, significant reduction in the spectral complexity, and suppressed light-scattering interferences (Andrade-Eiroa et al. 2010). With an appropriately selected $\Delta \lambda$, the SyF spectrum may contain bands of all the fluorescent components of the sample, making the analysis much faster as compared to using the EEM. The main characteristics of the SyF spectra are determined by the $\Delta \lambda$ value. The SyF spectra recorded over the range of $\Delta \lambda$ values are known as the total synchronous fluorescence (TSyF) spectra (Patra and Mishra 2002).

Both the excitation-emission spectra and the synchronous fluorescence spectra were used in the studies of the antioxidant contents and antioxidant capacity of the food samples. EEM fluorescence spectroscopy coupled with multivariate regression was used for screening the antioxidant content and antioxidant capacity of coffee, peppermint extracts, and tomato pastes (Orzel and Daszykowski 2014; Orzel et al. 2015) and for quantifying quercetin and kaempferol in the paprika samples, with good results obtained for quercetin (MonagoMaraña et al. 2016). SyF spectra were used for determination of the phenolic acids and scopoletin in brandies (Žiak et al. 2014).

Apples and apple juices are important dietary sources of phenolics (Biedrzycka and Amarowicz 2008; Candrawinata et al. 2013). The consumption of apples and apple products might be linked to a reduced risk of several forms of cancer, cardiovascular disease, and asthma (Hyson 2011). Apple phenolics include hydroxybenzoic and hydroxycinnamic acids and their derivatives, flavonols, dihydrochalcones, anthocyanins, and monomeric and oligomeric flavanols (procyanidins) (Kalinowska et al. 2014). Flavonoids are especially important antioxidants, acting as reducing agents, hydrogen donors, singlet oxygen quenchers, and metal chelating agents (Ignat et al. 2011).

NIR spectroscopy combined with multivariate regression was used to predict the antioxidant capacity and the 
polyphenolic content of apples (Giovanelli et al. 2014; Pissard et al. 2013; Schmutzler and Huck 2014; Schmutzler and Huck 2016).

The contribution of the polyphenolic compounds to the autofluorescence of apple juices was reported in studies of different aspects of the apple juice quality (Poryvkina et al. 2014; Seiden et al. 1996).

Recently, we reported a quantitative relationship between the EEMs of the apple juices and their phenolic contents and antioxidant capacity (Włodarska et al. 2016).

The aim of the present study is to investigate the feasibility of synchronous fluorescence coupled with chemometrics for screening the total phenolic content (TPC), total flavonoid content (TFC), and total antioxidant capacity (TAC) in commercial apple juices.

\section{Materials and Methods}

\section{Apple Juices}

Thirty commercial apple juices were evaluated in this study. The samples were available from a previous study and were labeled in the same way as in our previous publication (Włodarska et al. 2016). The capital letter denotes the sample group, the number denotes the producer, and the lowercase letters distinguish the different production batches. The studied samples include the juices reconstituted from concentrate, clear (group A-11 samples: A1a, A1b, A2, A3a, A3b, A4a, A4b, A5a, A5b, A6a, A6b) and cloudy (group B-2 samples: $\mathrm{B} 1$ and $\mathrm{B} 2$ ), and not from concentrate, including pasteurized naturally cloudy juices (group $\mathrm{C}-13$ samples: $\mathrm{C} 1 \mathrm{a}, \mathrm{C} 1 \mathrm{~b}$, C2a, C2b, C3a, C3b, C4a, C4b, C5a, C5b, C6a, C6b, and C7) and freshly squeezed juices (group D -4 samples: D1a, D1b, D2a, and D2b).

\section{Determination of Chemical Parameters}

The data on the TPC and the TAC of the studied juices were available from the previous study (Włodarska et al. 2016). Briefly, the TPC was determined according to Singleton and Rossi (1965) with modification for using 48-well microplates and expressed in milligrams of gallic acid equivalents per liter of juice (mg GAE/L).

The TAC of juices was determined by the Trolox equivalent antioxidant capacity (TEAC) assay according to Re et al. (1999), as described in detail in Gliszczyńska-Świgło et al. (2006). The TEAC value was expressed in millimoles of Trolox per liter of juice $(\mathrm{mM})$.

Total flavonoid content (TFC). The amount of flavonoids in the juices was determined according to Karadeniz et al. (2005) with modification for using 48 -well microplates. First, $0.10 \mathrm{~mL}$ of the juice sample was mixed with $0.50 \mathrm{~mL}$ of deionized water and $0.03 \mathrm{~mL}$ of $5 \%$ sodium nitrite (SigmaAldrich, Steinheim, Germany). After $5 \mathrm{~min}$ at room temperature, $0.06 \mathrm{~mL}$ of $10 \%$ aluminum chloride (Sigma-Aldrich, Steinheim, Germany) was added, and after $5 \mathrm{~min}, 0.20 \mathrm{~mL}$ of $1 \mathrm{M}$ sodium hydroxide (Sigma-Aldrich, Steinheim, Germany) was also added. Deionized water was used to adjust the total volume to $1 \mathrm{~mL}$. The absorbance of the samples was measured at $510 \mathrm{~nm}$ using an Epoch ${ }^{\mathrm{TM}}$ microplate spectrophotometer (BioTek). The TFC was reported as milligrams of catechin (Sigma-Aldrich, Steinheim, Germany) equivalents per liter of juice $(\mathrm{mg} \mathrm{CE} / \mathrm{L})$. These analyses were all made in triplicate.

\section{Fluorescence Measurements}

The fluorescence spectra were measured using a Fluorolog-311 spectrofluorometer (SPEX-Jobin Yvon, France). TSyF was obtained by measuring the SyF spectra in the $240-700 \mathrm{~nm}$ excitation range with the emission-excitation offsets $(\Delta \lambda)$ in the $10-160 \mathrm{~nm}$ range, with a $10 \mathrm{~nm}$ step. The individual SyF spectra were plotted as a function of the excitation wavelength.

The excitation and emission slit widths were $3 \mathrm{~nm}$. The acquisition interval and the integration time were maintained at $1 \mathrm{~nm}$ and $0.1 \mathrm{~s}$, respectively. A reference photodiode detector at the excitation monochromator stage compensated for the source intensity fluctuations. The individual spectra were corrected for the wavelength-dependent response of the system.

Front-face geometry was used for the undiluted samples in a 10-mm fused-silica cuvette. The samples of clear juices were measured directly, without any preparation; the samples of cloudy juices were measured after centrifugation $(15,000 \mathrm{rpm}$ for $5 \mathrm{~min})$.

\section{Data Analysis}

Pearson correlation coefficients were calculated to test for the correlations between the individual analytical parameters (Bower 2009).

Principal component analysis (PCA) was performed on the $X$ matrix that contains TSyF spectral data held in an unfolded array (Wold et al. 1987).

Regression analysis. Partial least squares (PLS) regression was used to establish the calibration models between the fluorescence data ( $X$ matrix) and the chemical parameters $(Y)$.

The PLS method models both the $X$ - and $Y$-matrices simultaneously, to find such latent variables in $X$ that will best predict the latent variables in $Y$ (Wold et al. 2001). Two PLS versions were used, PLS1 and PLS2. A PLS1 model enables to predict one $Y$ variable at a time, while a PLS2 model allows prediction of a number of $Y$ variables simultaneously (Wold et al. 2001). 
PLS was used to model the relation between the chemical data and two types of spectral data: unfolded array of the entire TSyF spectra and individual SyF spectra recorded at particular $\Delta \lambda$ offsets.

The $X$ data containing the entire TSyF were arranged for the analysis of the unfolded array into the matrix of $30 \times 7376$ elements (number of samples $\times$ number of $\Delta \lambda$ offsets $\times$ number of excitation wavelengths). The $X$ matrix for the analysis of the individual SyF spectra consisted of $30 \times 461$ elements (number of samples $\times$ number of excitation wavelengths). Thus, 16 individual models were developed for each of the chemical parameters using SyF spectra recorded at particular $\Delta \lambda$ offsets in the range from 10 to $160 \mathrm{~nm}$, with $10 \mathrm{~nm}$ increments.

PLS1 and PLS2 methods were used for the analysis of each of the spectral data arrangements. Individual models for each of the analytical parameters were evaluated using the PLS1 method; the $Y$ matrix consisted of $30 \times 1$ elements (number of samples $\times$ analytical parameter). The PLS2 method modeled all of the three analytical parameters simultaneously; the $Y$ matrix consisted of $30 \times 3$ elements (number of samples $\times$ analytical parameters). The data pre-treatment included mean centering.

Full leave-one-out cross-validation was applied to all of the regression models. The regression models were evaluated using the $R^{2}$ determination coefficient and the root mean square error of cross-validation (RMSECV). The optimal number of components was chosen as the minimum of the plot of the RMSECV in function of the number of components. The residual predictive deviation (RPD) was calculated as the ratio of the standard deviation of the response variables to the RMSECV (Nicolaï et al. 2007).

The data analysis was carried out using the Solo v. 5.0.1 software (Eigenvector Research Inc., USA).

\section{Results and Discussion}

\section{Synchronous Fluorescence Spectra of the Apple Juices}

Thirty apple juices studied were selected to cover the various ranges of juices available on the market: clear reconstituted from concentrate (group A), cloudy produced from concentrate with added apple pulp (group B), pasteurized naturally cloudy produced not from concentrate (group C), and freshly squeezed juices (group D). Figure 1 shows the TSyF of four apple juices that represent each of the categories studied.

Figure 1 illustrates the overall characteristics of the synchronous fluorescence spectra over the 10- to 160-nm range of the emission-excitation wavelength offsets $(\Delta \lambda)$. The dependence of the SyF spectra on $\Delta \lambda$ is quite clear. Three distinct emission zones were observed, with varied exact positions of the maxima and intensities in the spectra of different juices. The spectral maxima of these three zones appeared within the following $\Delta \lambda$ /excitation wavelength ranges: 40 100/260-280, 110-140/305-320, and 60-100/360-420 nm. This result corresponds to the presence of three groups of fluorophores with similar emission properties, observed in the excitation-emission matrices of the apple juices in our previous study (Włodarska et al. 2016).

One of the most important advantages of the synchronous fluorescence is an enhanced separation of the overlapping bands as compared to the conventional emission spectra (Andrade-Eiroa et al. 2010; Patra and Mishra 2002). This feature is expected for the spectra recorded at lower $\Delta \lambda$ values. In such conditions, the overlap zone of the absorption and emission is narrower, and the resulting spectra of the individual fluorophores are also narrower as compared to the conventionally recorded emission spectra (Patra and Mishra 2002). Therefore, the synchronous fluorescence spectra measured at selected $\Delta \lambda$ values are shown in Fig. 2 for a detailed analysis.

Several bands appeared in the spectra recorded at $\Delta \lambda=30 \mathrm{~nm}$, with different intensity, and the respective maxima at about 275-280 nm, 300-310 nm (low intensity), 365-370 nm, 440-460 nm (low intensity), 475$485 \mathrm{~nm}$, and 655-660 nm (low intensity). This observation shows that the emission of the apple juices is in fact quite complex, with more than the initially identified three groups of fluorophores present in the samples studied. Both the intensity and the spectral width increased with increasing $\Delta \lambda$, with the maxima shifting to shorter wavelengths. These changes lead to an increase in the spectral overlapping observed at higher $\Delta \lambda$. The intensity of the individual emission bands first reached maximum at a particular $\Delta \lambda$ value, decreasing again with the further increase of the offset value. It should be noted that the different fluorophores achieve their maximum intensity at different $\Delta \lambda$ values; therefore, the relative intensities of the particular bands in the SyF spectra also varied with $\Delta \lambda$.

PCA analysis was performed on the unfolded TSyF spectra of all of the studied juices for a comparison of the fluorescence patterns of the entire sample set (Fig. 3).

The juices from the different groups were to some extent discriminated by their PC1 and PC2 components, explaining respectively 80 and $10 \%$ of the spectral variation. $\mathrm{PC} 1$, which represents the main direction of the spectral variability, is negatively correlated with the emission below $300 \mathrm{~nm}$ and positively with the emission in the 350-450-nm range. The juices of the $\mathrm{C}$ group showed the most diverse fluorescence, spreading widely along both PC1 and PC2 axes.

The native apple juice fluorescence may originate from several chemical components. The phenolics present in the apple juices include chlorogenic acid, caffeic acid, pcoumaric acid, ferulic acid, catechin, epicatechin, procyanidines (B1, B2, C1 trimer), rutin, phloridzin, 
Fig. 1. Total synchronous fluorescence (TSyF) spectra of the apple juices: a clear from concentrate (sample A1a; $\mathrm{TPC}=198.4 \mathrm{mg} \mathrm{GAE} / \mathrm{L}$, $\mathrm{TFC}=52.7 \mathrm{mg} \mathrm{CE} / \mathrm{L}$,

$\mathrm{TAC}=1.06 \mathrm{mM}), \mathbf{b}$ cloudy from concentrate (sample B2;

$\mathrm{TPC}=386.5 \mathrm{mg} \mathrm{GAE} / \mathrm{L}$,

$\mathrm{TFC}=153.4 \mathrm{mg} \mathrm{CE} / \mathrm{L}$,

$\mathrm{TAC}=4.43 \mathrm{mM})$, c cloudy not from concentrate (sample $\mathrm{C} 5 \mathrm{a}$; $\mathrm{TPC}=174.2 \mathrm{mg} \mathrm{GAE} / \mathrm{L}$,

$\mathrm{TFC}=54.7 \mathrm{mg} \mathrm{CE} / \mathrm{L}$,

$\mathrm{TAC}=1.36 \mathrm{mM})$, and $\mathbf{d}$ freshly squeezed (sample D2a;

$\mathrm{TPC}=391.2 \mathrm{mg} \mathrm{GAE} / \mathrm{L}$,

$\mathrm{TFC}=42.2 \mathrm{mg} \mathrm{CE} / \mathrm{L}$

$\mathrm{TAC}=3.05 \mathrm{mM}$ )

Fig. 2. Synchronous

fluorescence (SyF) spectra of the apple juices recorded at different $\Delta \lambda$ offsets: a $30 \mathrm{~nm}, \mathbf{b} 80 \mathrm{~nm}, \mathbf{c}$ $120 \mathrm{~nm}$, and d $160 \mathrm{~nm}$. Samples: clear from concentrate (sample A1a), cloudy from concentrate (sample B2), cloudy not from concentrate (sample C5a), and freshly squeezed (sample D2a)
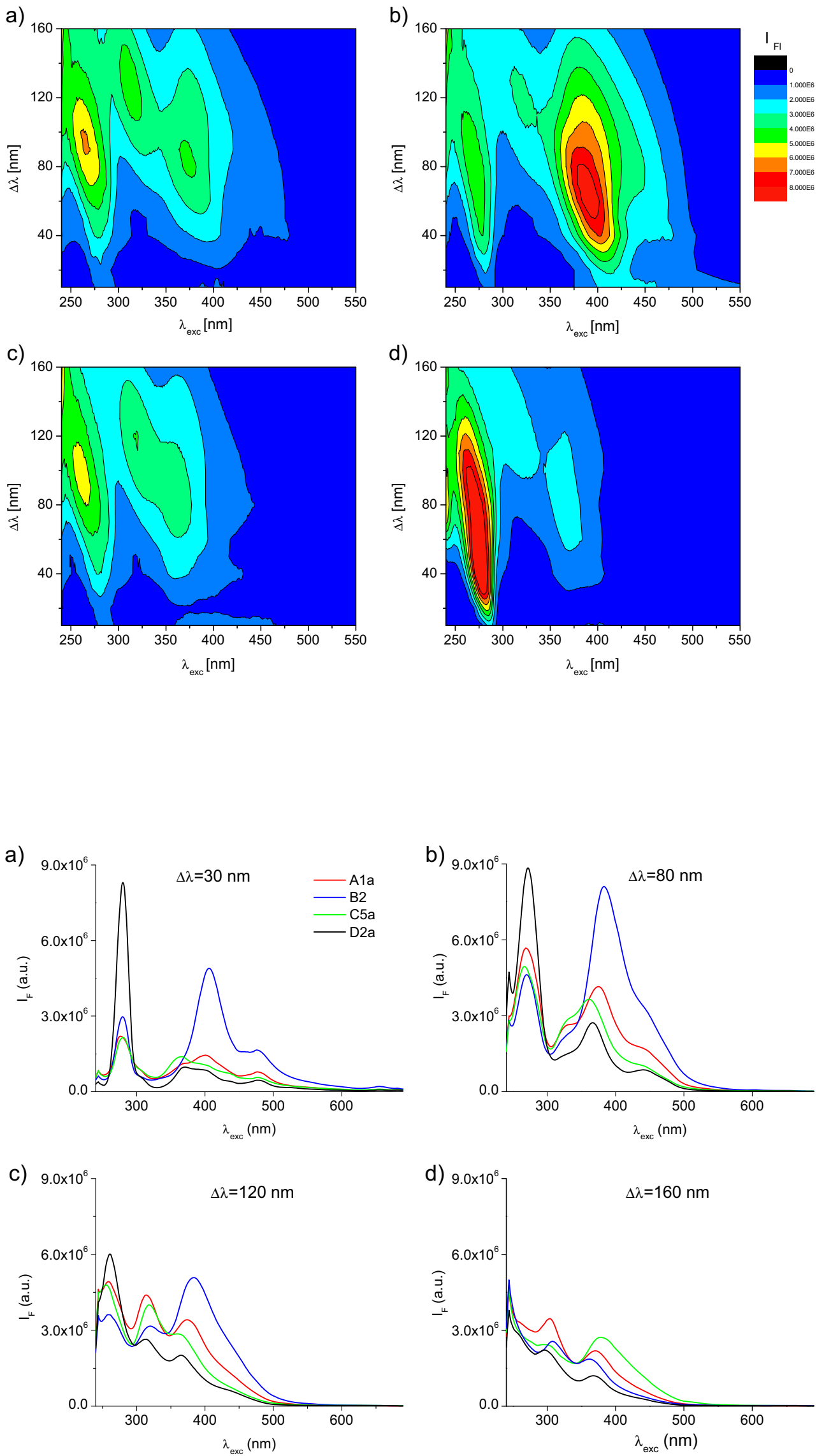
a)

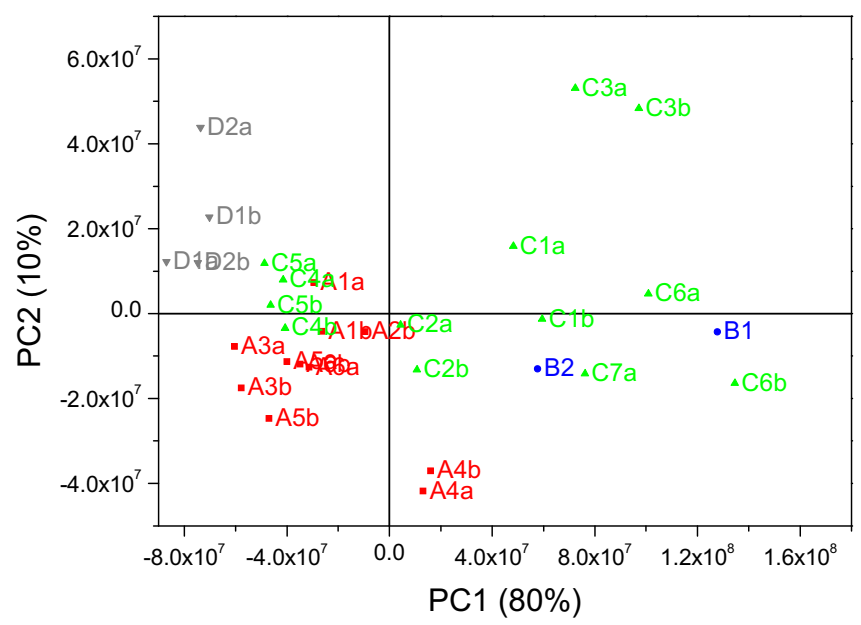

Fig. 3. The principal component analysis of the unfolded total synchronous fluorescence (TSyF) spectra of all the apple juices studied: a score plot, b loading plot for PC1. Four groups of samples: A (red)-

quercetin, isoquercitrin, and hyperin (Gliszczyńska-Świgło and Tyrakowska 2003; Kahle et al. 2005).

Chlorogenic acid, the main phenolic component of the apple juices, has a single maximum at approximately $337 \mathrm{~nm}$ in SyF recorded at $\Delta \lambda=110 \mathrm{~nm}$ in methanolic solution (Morales et al. 2005). The emission was observed in the following ranges of $\Delta \lambda$ /excitation wavelength values in the TSyF spectra of the phenolic standards recorded in methanol: 90-110/ 355-365 $\mathrm{nm}$ for chlorogenic acid, 80-95/365-375 nm for caffeic acid, 60-95/355-365 $\mathrm{nm}$ for ferulic acid, and 50-95/ 410-435 nm for kaempferol (Sergiel et al. 2014). The SyF spectra of catechin and epicatechin, to our best knowledge, have not been reported previously. Based on excitation and emission properties of these compounds, they should rather contribute to the short-wavelength emission in the SyF spectra (Airado-Rodríguez et al. 2011).

Other compounds beside phenolics may also contribute to the apple juice fluorescence. The fluorescence of products of the non-enzymatic browning of the apple juice is well documented in literature (Cohen et al. 1998; Zhu et al. 2009). The presence of other fluorescent compounds including aromatic amino acids (tyrosine and phenylalanine) and water-soluble vitamins $\mathrm{B}_{2}$ and $\mathrm{B}_{6}$ was also reported in apples (Christensen et al. 2006; Feliciano et al. 2010; $\mathrm{Wu}$ et al. 2007). Based on the published data, the emission of vitamins $B_{2}$ and $B_{6}$ and of the products of the non-enzymatic browning should be expected rather in the intermediate range, while the aromatic amino acids should emit in the short-wavelength range (Airado-Rodríguez et al. 2011; Christensen et al. 2006). The longerwavelength band with a very low intensity with the maximum at $655 \mathrm{~nm}$ corresponds to chlorophyll (Christensen et al. 2006). b)

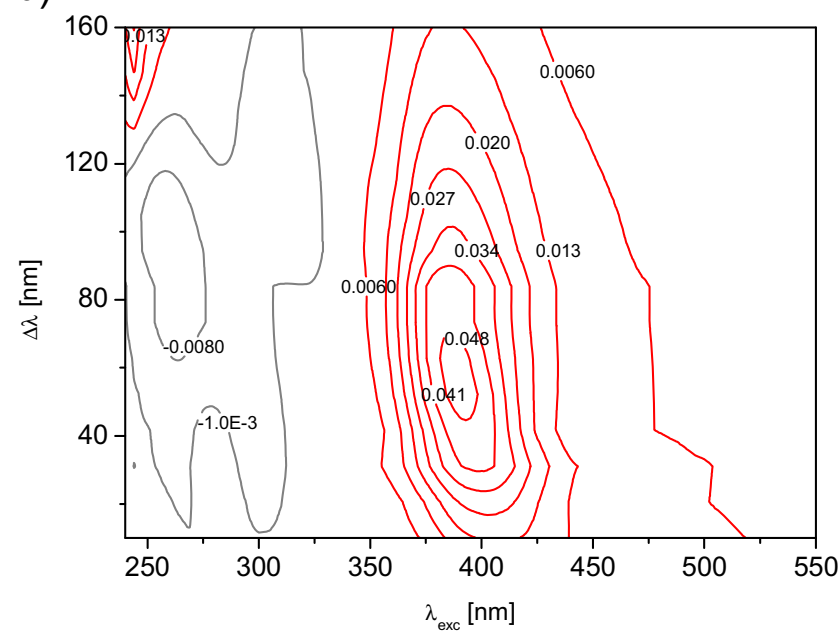

clear from concentrate, $B$ (blue) — cloudy from concentrate, $C$ (green) cloudy not from concentrate, $D$ (gray) - freshly squeezed. (Color figure online)

It should be noted that the fluorescence is sensitive to the micro-environment, solvent polarity, and $\mathrm{pH}$ values; therefore, the spectra of the standard solutions may differ from the emission of the same component recorded in the food matrix (Christensen et al. 2006).

\section{Multivariate Calibration Models}

Chemical Characteristics of the Calibration Set All of the samples $(n=30)$ were used in the analysis, with the chemical characteristics of the calibration set shown in Table 1. The mean values and standard deviations of the TPC, TFC, and TAC of the studied samples are also listed in Table 1.

The present results confirmed variability of the antioxidant properties of apple juices studied (Włodarska et al. 2016). The TFC determined by the spectrophotometric method was in the range of 42.23-298.7 $\mathrm{mg} \mathrm{CE} / \mathrm{L}$, with the mean value and standard deviation of $134 \pm 69$. It is well known that the contents and profiles of the phenolic compounds in apple juices vary depending on the raw material and processing conditions (Candrawinata et al. 2013; Kalinowska et al. 2014; Markowski et al. 2015; Oszmiański et al. 2009).

Significant positive correlations were observed between the three studied analytical parameters: TPC and TAC $(r=0.944)$ (Włodarska et al. 2016), TFC and TAC $(r=0.679)$, and TPC and TFC $(r=0.741)$. These results confirm that the antioxidant properties of the apple juices are determined by their phenolic contents, in agreement with the published data (Gardner et al. 2000; Gliszczyńska-Świgło and Tyrakowska 2003). In some of the apple juices studied, the producers declared added vitamin $\mathrm{C}$, which may also contribute to the antioxidant properties (Włodarska et al. 2016). 
Table 1 Characteristics of the regression models for the prediction of the total phenolic (TPC) and flavonoid (TFC) contents and the antioxidant activity (TAC) of the apple juices ( $n=30$ samples) using synchronous fluorescence spectra

\begin{tabular}{|c|c|c|c|c|c|c|}
\hline PLS model & LV & $\begin{array}{l}\text { PLS } \\
R^{2}\end{array}$ & $\begin{array}{l}\text { calibration } \\
\text { RMSEC }\end{array}$ & $\begin{array}{l}\text { PLS } \\
R^{2}\end{array}$ & $\begin{array}{l}\text { cross-validation } \\
\text { RMSECV }\end{array}$ & RPD \\
\hline \multicolumn{7}{|c|}{$\mathrm{TPC} ;(552 \pm 403) \mathrm{mg} \mathrm{GAE} / \mathrm{L}$} \\
\hline TSyF unfolded PLS 1 & 9 & 0.970 & 68.9 & 0.740 & 208.7 & 1.9 \\
\hline TSyF unfolded PLS 2 & 9 & 0.969 & 69.2 & 0.744 & 207.2 & 1.9 \\
\hline SyF PLS $1 \Delta \lambda=80 \mathrm{~nm}$ & 11 & 0.968 & 70.7 & 0.766 & 198.0 & 2.0 \\
\hline SyF PLS $2 \Delta \lambda=80 \mathrm{~nm}$ & 11 & 0.968 & 70.8 & 0.766 & 198.0 & 2.0 \\
\hline \multicolumn{7}{|c|}{ TFC; $(134 \pm 69) \mathrm{mg} \mathrm{CE} / \mathrm{L}$} \\
\hline TSyF unfolded PLS 1 & 3 & 0.901 & 21.4 & 0.846 & 27.6 & 2.5 \\
\hline TSyF unfolded PLS 2 & 9 & 0.921 & 19.1 & 0.824 & 29.5 & 2.3 \\
\hline SyF PLS $1 \Delta \lambda=110 \mathrm{~nm}$ & 2 & 0.888 & 22.8 & 0.870 & 25.3 & 2.7 \\
\hline SyF PLS $2 \Delta \lambda=110 \mathrm{~nm}$ & 2 & 0.873 & 24.2 & 0.857 & 26.6 & 2.6 \\
\hline \multicolumn{7}{|c|}{$\mathrm{TAC} ;(4.6 \pm 3.8) \mathrm{mM}$} \\
\hline TSyF unfolded PLS 1 & 9 & 0.962 & 0.73 & 0.708 & 2.11 & 1.8 \\
\hline TSyF unfolded PLS 2 & 9 & 0.929 & 1.01 & 0.765 & 1.89 & 2.0 \\
\hline SyF PLS $1 \Delta \lambda=70 \mathrm{~nm}$ & 11 & 0.976 & 0.59 & 0.787 & 1.80 & 2.1 \\
\hline SyF PLS $2 \Delta \lambda=70 \mathrm{~nm}$ & 10 & 0.926 & 1.03 & 0.709 & 2.11 & 1.8 \\
\hline
\end{tabular}

$L V$ number of latent variables used for PLS, $R^{2}$ determination coefficient, $R M S E C V$ root mean square errors of calibration and cross-validation in original units (mg GAE/L for TPC, mg CE/L for TFC, mM for TAC), RPD residual predictive deviation
Regression Analysis Multivariate regression was used in order to model the relationship between the fluorescence and the analytical characteristics (TPC, TFC, and TAC). The regression using PLS was performed for the analysis of the unfolded TSyF and single-offset SyF spectra. Two versions of the regression method were used: PLS1 that models each of the analytical parameters individually and PLS2 that analyzes all of the parameters simultaneously. The pre-processing methods tested (smoothing and first derivative) provided no pronounced improvement in the regression results. Meancentered spectra were used in the regression analysis.

Table 1 presents the results of the multivariate calibrations. All of the calibration models confirm the quantitative relationship between the SyF spectra and the analytical parameters studied. The best models were obtained for the prediction of TFC using almost all of the regression methods. The models for TPC and TAC had generally higher prediction errors and lower $R^{2}$ and RPD values, as compared to the models for TFC.

Some improvements in the model performance were observed for the analysis of the single-offset SyF as compared to the unfolded TSyF. The characteristics of the PLS1 models were in most cases similar to those of the respective PLS2 models.

When the regression analysis was applied to the singleoffset SyF spectra, the model performance varied in function of the $\Delta \lambda$ value at which the particular spectra were recorded (Fig. 4).

The best models for the TPC were obtained using the SyF spectra recorded at $\Delta \lambda=50$ and $70-100 \mathrm{~nm}$. The optimal regression model for the TPC was obtained using the SyF spectrum recorded at $\Delta \lambda=80 \mathrm{~nm}$, characterized by $\mathrm{RPD}=2.0$ that corresponds to an approximate prediction ability (Nicolaï et al. 2007).

The regression models for the TFC had generally better performance as compared to those for the other two parameters, TPC and TAC. The spectral analysis at $\Delta \lambda=100$ $160 \mathrm{~nm}$, with the exception of $140 \mathrm{~nm}$, gave models with better parameters ( $\mathrm{RPD}=2.5-2.6)$. The model with the best predictive ability for the TFC $(\mathrm{RPD}=2.7)$ was obtained for the SyF spectrum at $\Delta \lambda=110 \mathrm{~nm}$.

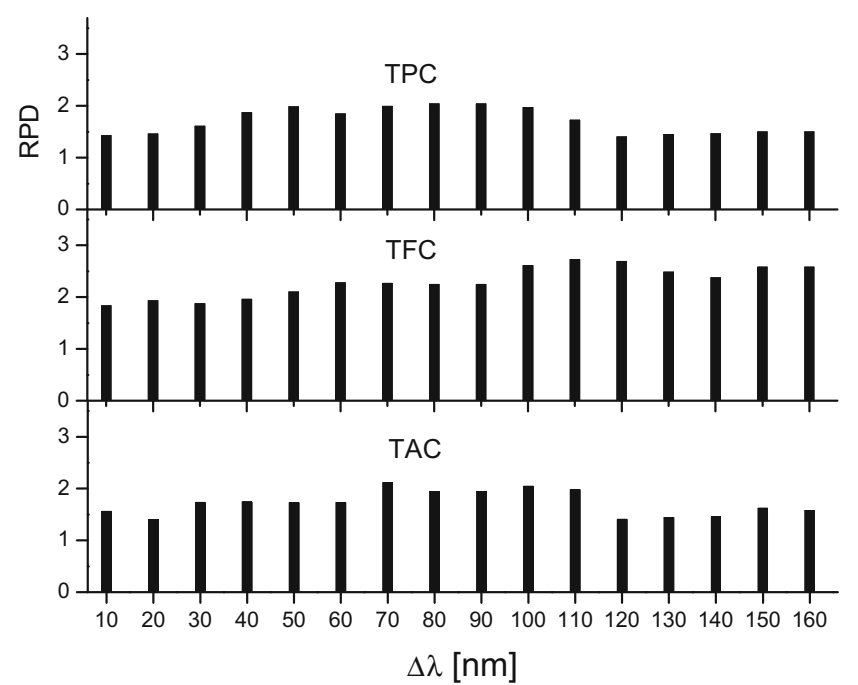

Fig. 4. Dependence of the relative predictive deviation (RPD) on the $\Delta \lambda$ offset for the PLS1 calibration models for the prediction of the total flavonoid content (TFC), the total phenolic content (TPC), and the total antioxidant capacity (TAC) 
The regression models for TAC using the SyF spectra at $\Delta \lambda=70-110 \mathrm{~nm}$ had better performance. The best model was obtained for the spectra at $\Delta \lambda=70 \mathrm{~nm}(\mathrm{RPD}=2.1)$.

The regression models for the TPC and TAC include higher numbers of latent variables as compared to the models for $\mathrm{TFC}$, in line with the better performance of the latter. It seems that the better performance of the TFC calibration models confirms the meaningful contribution of the flavonoids to the apple juice fluorescence.

The presently reported models based on the SyF spectra have only slightly lower performance as compared to those based on EEM. The respective parameters of the models obtained using EEM were $R^{2} \mathrm{CV}=0.802, \mathrm{RPD}=2.3$ for the TPC, and $R_{\mathrm{CV}}^{2}=0.808, \mathrm{RPD}=2.3$ for the TAC (Włodarska et al. 2016).

The predictive ability of the models based both on the SyF and EEM, as judged by the RPD criterion, corresponds to the approximate quantitative prediction accuracy (Nicolaï et al. 2007). However, the important advantages of the SyF spectra over the EEM are the much faster measurements and the simpler data analysis. Therefore, it could be more favorable to use synchronous fluorescence in the screening analysis.

Prediction of the New Samples To test the model performance for prediction of the unknown samples, five new apple juices were used from the same producers but different production batches (Table 2).

The optimal calibration model for each of the parameters was used to predict TPC, TFC, and TAC. The best results were obtained for the prediction of TFC, with the average relative error of $18 \%$. The relative error was equal to $19 \%$ for TAC; in contrast, TPC was predicted with a considerably higher average error of $31 \%$. However, the relative errors for TPC and TAC prediction based on individual samples showed relatively wide ranges (Table 2), indicating lower stability and confirming the generally lower performance of the models for the prediction of these two parameters.
Taking into account both the results of the cross-validation and prediction of the new samples, we conclude that the models are quite appropriate only for the semi-quantitative and screening analysis. The model for the prediction of TFC had good predictive ability, while those for TPC and TAC have approximate predictive ability.

To the best of our knowledge, there are no reports on the prediction of antioxidant properties in apple juices using other spectroscopic techniques. Very variable RPD values were reported for the determination of TPC in the analysis of intact apples using NIR spectroscopy: 5.1 (Pissard et al. 2013), 1.25-1.87 (Schmutzler and Huck 2014), and 3.6 (Giovanelli et al. 2014). The RPD values of 2.8 (Schmutzler and Huck 2016) and 3.0 (Giovanelli et al. 2014) were reported for the prediction of TAC using the same method.

The observed inferior performance of the models based on fluorescence for precise quantitative analysis may have several explanations, as we discussed recently (Włodarska et al. 2016). Firstly, different compounds, both fluorescent and non-fluorescent, may contribute to the antioxidant activity of juices. Although the main phenolic compound present in apple juices is the fluorescent chlorogenic acid, a wide range of other phenolic compounds (Gliszczyńska-Świgło and Tyrakowska 2003; Kahle et al. 2005) with very diverse fluorescence properties (Mazina et al. 2015; Wolfbeis et al. 1984) was reported in these products. The overall fluorescence of a juice depends not only on the concentrations of the individual fluorescent components but also on their fluorescence quantum yields (Christensen et al. 2006). Moreover, the fluorescence may be affected by the sample matrix properties.

The Folin-Ciocalteu assay used for the determination of TPC has a limited specificity; thus, non-phenolic compounds may also contribute to the result (Craft et al. 2012). In particular, the presence of vitamin $\mathrm{C}$ may affect the TPC assay. The addition of vitamin $\mathrm{C}$ to some of the juices studied was declared by the respective producers (Włodarska et al. 2016). Similarly, the method used for TAC determination is sensitive for a range of fluorescent and non-fluorescent compounds

Table 2 Prediction results of the total phenolic content (TPC), total flavonoid content (TFC), and total antioxidant capacity (TAC) in unknown samples, using the optimal regression models for each of the parameters

\begin{tabular}{|c|c|c|c|c|c|c|c|c|c|}
\hline $\begin{array}{l}\text { PLS model } \\
\text { Sample }\end{array}$ & $\begin{array}{l}\text { TPC } \\
\text { Predicted } \\
(\mathrm{mg} \mathrm{GAE} / \mathrm{L})\end{array}$ & $\begin{array}{l}\Delta \lambda=80 \mathrm{~nm} \\
\text { Reference } \\
(\mathrm{mg} \text { GAE } / \mathrm{L})\end{array}$ & $\begin{array}{l}\text { Relative } \\
\text { error }^{\mathrm{a}}(\%)\end{array}$ & $\begin{array}{l}\text { TFC } \\
\text { Predicted } \\
(\mathrm{mg} \mathrm{CE} / \mathrm{L})\end{array}$ & $\begin{array}{l}\Delta \lambda=110 \mathrm{~nm} \\
\text { Reference } \\
(\mathrm{mg} C E / \mathrm{L})\end{array}$ & $\begin{array}{l}\text { Relative } \\
\text { error }^{\mathrm{a}}(\%)\end{array}$ & $\begin{array}{l}\text { TAC } \\
\text { Predicted } \\
(\mathrm{mM})\end{array}$ & $\begin{array}{l}\Delta \lambda=70 \mathrm{~nm} \\
\text { Reference } \\
(\mathrm{mM})\end{array}$ & $\begin{array}{l}\text { Relative } \\
\text { error }^{\mathrm{a}}(\%)\end{array}$ \\
\hline 1 & 849 & 640 & 33 & 194 & 251 & 23 & 8.3 & 6.2 & 34 \\
\hline 2 & 334 & 359 & 7 & 103 & 123 & 16 & 1.6 & 2.7 & 41 \\
\hline 3 & 1084 & 761 & 42 & 211 & 242 & 13 & 9.1 & 8.4 & 8 \\
\hline 4 & 613 & 447 & 37 & 185 & 157 & 18 & 4.9 & 4.6 & 7 \\
\hline 5 & 570 & 425 & 34 & 100 & 128 & 22 & 3.7 & 3.9 & 5 \\
\hline Average error (\%) & & & 31 & & & 18 & & & 19 \\
\hline
\end{tabular}

${ }^{a}$ Relative errors were calculated as $(\mid$ Reference - Predicted $\mid /$ Reference $) \times 100 \%$ 
with antioxidant properties. Interestingly, better results were obtained for modeling TFC as compared to TPC and TAC. This may indicate that fluorescence may be better suited for the analysis of a selected group of compounds or even of individual fluorescent antioxidants instead of the TPC or TAC determination; however, this hypothesis needs further testing.

\section{Conclusions}

The apple juices showed some characteristic fluorescence patterns common to all of the studied samples, although the spectra of the individual juices had very diverse characteristics. Synchronous fluorescence spectroscopy revealed several additional bands originating from different fluorophores, as compared to EEM, due to the reduced spectral overlapping in SyF.

The antioxidant properties of the apple juices could be modeled using their SyF spectra. The regression models based on single-offset SyF spectra showed better performance than those based on the entire TSyF spectra. The dependence of the SyF on the $\Delta \lambda$ offset should be considered in order to optimize the analytic parameters. The spectra recorded at lower $\Delta \lambda$ provide better resolution for the qualitative analysis. However, the optimal regression models were obtained at higher $\Delta \lambda$ values $(\Delta \lambda=70-110 \mathrm{~nm})$.

We have to underline that the models obtained were only appropriate for the semi-quantitative analysis. The model for TFC had the best performance. The predictive ability of the PLS models based on SyF and EEM was similar. Therefore, the single-offset SyF is preferable for the screening analysis, instead of the entire EEM. This may significantly shorten the recording time and simplify the analysis, due to the reduced amount of spectral data.

In summary, we conclude that modeling of antioxidant properties based on fluorescence is quite a complex task; therefore, further studies are needed to evaluate the feasibility of this method for precise quantitative analysis.

Acknowledgments We thank Prof. Anna Gliszczyńska-Świgło for the protocols for determining the analytical parameters according to the reference methods.

\section{Compliance with Ethical Standards}

Conflict of Interest Katarzyna Włodarska declares that she has no conflict of interest. Katarzyna Pawlak-Lemańska declares that she has no conflict of interest. Igor Khmelinskii declares that he has no conflict of interest. Ewa Sikorska declares that she has no conflicts of interest.

Ethical Approval This article does not contain any studies with human participants or animals performed by any of the authors.

Informed Consent Not applicable.
Open Access This article is distributed under the terms of the Creative Commons Attribution 4.0 International License (http:// creativecommons.org/licenses/by/4.0/), which permits unrestricted use, distribution, and reproduction in any medium, provided you give appropriate credit to the original author(s) and the source, provide a link to the Creative Commons license, and indicate if changes were made.

\section{References}

Airado-Rodríguez D, Durán-Merás I, Galeano-Díaz T, Wold JP (2011) Front-face fluorescence spectroscopy: a new tool for control in the wine industry. J Food Compos Anal 24:257-264

Andrade-Eiroa Á, de-Armas G, Estela J-M, Cerdà V (2010) Critical approach to synchronous spectrofluorimetry. I. TrAC Trends Anal Chem 29:885-901

Armenta S, Garrigues S, de la Guardia M (2008) Green analytical chemistry. TrAC-Trend Anal Chem 27:497-511

Biedrzycka E, Amarowicz R (2008) Diet and health: apple polyphenols as antioxidants. Food Rev Int 24:235-251

Bower JA (2009) Association, correlation and regression. In statistical methods for food science. Wiley, Chichester, pp. 137-149

Candrawinata VI, Golding JB, Roach PD, Stathopoulos CE (2013) From apple to juice - the fate of polyphenolic compounds. Food Rev Int 29:276-293

Christensen J, Nørgaard L, Bro R, Engelsen SB (2006) Multivariate autofluorescence of intact food systems. Chem Rev 106:1979-1994

Cohen E, Birk Y, Mannheim CH, Saguy IS (1998) A rapid method to monitor quality of apple juice during thermal processing. LWT-Food Sci Technol 31:612-616

Corradini E, Foglia P, Giansanti P, Gubbiotti R, Samperi R, Laganà A (2011) Flavonoids: chemical properties and analytical methodologies of identification and quantitation in foods and plants. Nat Prod Res 25:469-495

Cozzolino D (2015) Infrared spectroscopy as a versatile analytical tool for the quantitative determination of antioxidants in agricultural products, foods and plants. Antioxidants 4:482

Craft BD, Kerrihard AL, Amarowicz R, Pegg RB (2012) Phenol-based antioxidants and the in vitro methods used for their assessment. Compr Rev Food Sci F 11:148-173

Feliciano RP, Antunes C, Ramos A, Serra A. T, Figueira ME, Duarte CMM., de Carvalho A, Bronze MR (2010) Characterization of traditional and exotic apple varieties from Portugal. Part $1-$ nutritional, phytochemical and sensory evaluation. J Funct Foods 2:35-45

Gardner PT, White TAC, McPhail DB, Duthie GG (2000) The relative contributions of vitamin $\mathrm{C}$, carotenoids and phenolics to the antioxidant potential of fruit juices. Food Chem 68:471-474

Giovanelli G, Sinelli N, Beghi R, Guidetti R, Casiraghi E (2014) NIR spectroscopy for the optimization of postharvest apple management. Postharvest Biol Tec 87:13-20

Gliszczyńska-Świgło A, Tyrakowska B (2003) Quality of commercial apple juices evaluated on the basis of the polyphenol content and the TEAC antioxidant activity. J Food Sci 68:1844-1849

Gliszczyńska-Świgło A, Ciska E, Pawlak-Lemańska K, Chmielewski J, Borkowski T, Tyrakowska B (2006) Changes in the content of health-promoting compounds and antioxidant activity of broccoli after domestic processing. Food Addit Contam 23:1088-1098

Hyson DA (2011) A comprehensive review of apples and apple components and their relationship to human health. Adv Nutr 2:408-420

Ignat I, Volf I, Popa VI (2011) A critical review of methods for characterisation of polyphenolic compounds in fruits and vegetables. Food Chem 126:1821-1835

Kahle K, Kraus M, Richling E (2005) Polyphenol profiles of apple juices. Mol Nut Food Res 49:797-806

Kalinowska M, Bielawska A, Lewandowska-Siwkiewicz H, Priebe W, Lewandowski W (2014) Apples: content of phenolic compounds vs. 
variety, part of apple and cultivation model, extraction of phenolic compounds, biological properties. Plant Physiol Bioch 84:169-188

Karadeniz F, Burdurlu HS, Koca N, Soyer Y (2005) Antioxidant activity of selected fruits and vegetables grown in Turkey. Turk J Agric For 29:297-303

Lohumi S, Lee S, Lee H, Cho B-K (2015) A review of vibrational spectroscopic techniques for the detection of food authenticity and adulteration. Trends Food Sci Tech 46:85-98

$\mathrm{Lu}$ X, Rasco BA (2011) Determination of antioxidant content and antioxidant activity in foods using infrared spectroscopy and chemometrics: a review. Crit Rev Food Sci 52:853-875

Markowski J, Baron A, Le Quere J-M, Płocharski W (2015) Composition of clear and cloudy juices from French and Polish apples in relation to processing technology. LWT-Food Sci Technol 62:813-820

Mazina J, Vaher M, Kuhtinskaja M, Poryvkina L, Kaljurand M (2015) Fluorescence, electrophoretic and chromatographic fingerprints of herbal medicines and their comparative chemometric analysis. Talanta 139:233-246

Monago-Maraña O, Durán-Merás I, Galeano-Díaz T, Peña AM (2016) Fluorescence properties of flavonoid compounds. Quantification in paprika samples using spectrofluorimetry coupled to second order chemometric tools. Food Chem 196:1058-1065

Morales F, Cartelat A, Álvarez-Fernández A, Moya I, Cerovic ZG (2005) Time-resolved spectral studies of blue-green fluorescence of artichoke (Cynara cardunculus L. Var. Scolymus) leaves: identification of chlorogenic acid as one of the major fluorophores and agemediated changes. J Agr Food Chem 53:9668-9678

Nicolaï BM, Beullens K, Bobelyn E, Peirs A, Saeys W, Theron KI, Lammertyn J (2007) Nondestructive measurement of fruit and vegetable quality by means of NIR spectroscopy: a review. Postharvest Biol Tec 46:99-118

Orzel J, Daszykowski M (2014) A rapid validation of the antioxidant capacity of food commodities based on their fluorescence excitation emission spectra as applicable to coffee and peppermint extracts. Chemometr Intell Lab 137:74-81

Orzel J, Stanimirova I, Czarnik-Matusewicz B, Daszykowski M (2015) Prediction of the hydrophilic antioxidant capacity of tomato pastes from the IR and fluorescence excitation-emission spectra of extracts and intact samples. Talanta 138:64-70

Oszmiański J, Wojdylo A, Kolniak J (2009) Effect of enzymatic mash treatment and storage on phenolic composition, antioxidant activity, and turbidity of cloudy apple juice. J Agr Food Chem 57:7078-7085

Patra D, Mishra AK (2002) Recent developments in multi-component synchronous fluorescence scan analysis. TrAC-Trend Anal Chem 21:787-798

Pissard A, Fernández Pierna JA, Baeten V, Sinnaeve G, Lognay G, Mouteau A, Dupont P, Rondia A, Lateur M (2013) Nondestructive measurement of vitamin $\mathrm{C}$, total polyphenol and sugar content in apples using near-infrared spectroscopy. J Sci Food Agr $93: 238-244$
Poryvkina L, Tsvetkova N, Sobolev I (2014) Evaluation of apple juice quality using spectral fluorescence signatures. Food Chem 152:573577

Pyrzynska K, Sentkowska A (2014) Recent developments in the HPLC separation of phenolic food compounds. Crit Rev Anal Chem 45: $41-51$

Re R, Pellegrini N, Proteggente A, Pannala A, Yang M, Rice-Evans C (1999) Antioxidant activity applying an improved ABTS radical cation decolorization assay. Free Radical Bio Med 26:1231-1237

Robbins RJ (2003) Phenolic acids in foods: an overview of analytical methodology. J Agr Food Chem 51:2866-2887

Scalbert A, Manach C, Morand C, Rémésy C, Jiménez L (2005) Dietary polyphenols and the prevention of diseases. Crit Rev Food Sci 45: 287-306

Schmutzler M, Huck CW (2014) Automatic sample rotation for simultaneous determination of geographical origin and quality characteristics of apples based on near infrared spectroscopy (NIRS). Vib Spectrosc 72:97-104

Schmutzler M, Huck CW (2016) Simultaneous detection of total antioxidant capacity and total soluble solids content by Fourier transform near-infrared (FT-NIR) spectroscopy: a quick and sensitive method for on-site analyses of apples. Food Control 66:27-37

Seiden P, Bro R, Poll L, Munck L (1996) Exploring fluorescence spectra of apple juice and their connection to quality parameters by chemometrics. J Agr Food Chem 44:3202-3205

Sergiel I, Pohl P, Biesaga M, Mironczyk A (2014) Suitability of threedimensional synchronous fluorescence spectroscopy for fingerprint analysis of honey samples with reference to their phenolic profiles. Food Chem 145:319-326

Singleton VL, Rossi JA (1965) Colorimetry of total phenolics with phosphomolybdic-phosphotungstic acid reagents. Am J Enol Viticult 16:144-158

Włodarska K, Pawlak-Lemańska K, Khmelinskii I, Sikorska E (2016) Explorative study of apple juice fluorescence in relation to antioxidant properties. Food Chem 210:593-599

Wold S, Esbensen K, Geladi P (1987) Principal component analysis. Chemometr Intell Lab 2:37-52

Wold S, Sjöström M, Eriksson L (2001) PLS-regression: a basic tool of chemometrics. Chemometr Intell Lab 58:109-130

Wolfbeis OS, Begum M, Geiger H (1984) Fluorescence properties of hydroxy- and methoxyflavones and the effect of shift reagents. $Z$ Naturforsch 39b:231-237

Wu J, Gao H, Zhao L, Liao X, Chen F, Wang Z, Hu X (2007) Chemical compositional characterization of some apple cultivars. Food Chem 103:88-93

Zhu D, Ji B, Eum HL, Zude M (2009) Evaluation of the non-enzymatic browning in thermally processed apple juice by front-face fluorescence spectroscopy. Food Chem 113:272-279

Žiak L, Sádecká J, Májek P, Hroboňová K (2014) Simultaneous determination of phenolic acids and scopoletin in brandies using synchronous fluorescence spectrometry coupled with partial least squares. Food Anal Method 7:563-570 